\title{
The Danish Microbiology Database (MiBa) 2010 to 2013
}

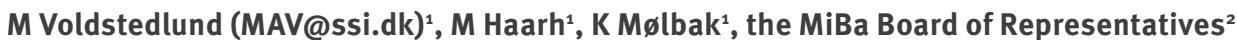

1. Department of Infectious Disease Epidemiology, Statens Serum Institute, Copenhagen, Denmark

2. The representatives of the board are listed at the end of the article

Citation style for this article:

Voldstedlund M, Haarh M, Mølbak K, the MiBa Board of Representatives. The Danish Microbiology Database (MiBa) 2010 to 2013 . Euro Surveill.

2014;19(1): pii=20667. Available online: http://www.eurosurveillance.org/ViewArticle.aspx?Articleld=20667

Article submitted on 08 July 2013 / published on 09 January 2014

The Danish Microbiology Database $(\mathrm{MiBa})$ is a national database that receives copies of reports from all Danish departments of clinical microbiology. The database was launched in order to provide healthcare personnel with nationwide access to microbiology reports and to enable real-time surveillance of communicable diseases and microorganisms. The establishment and management of MiBa has been a collaborative process among stakeholders, and the present paper summarises lessons learned from this nationwide endeavour which may be relevant to similar projects in the rapidly changing landscape of health informatics.

\section{Background}

In Denmark, surveillance of infectious diseases is based on notifications from physicians in hospitals and general practices as well as statutory reporting from departments of clinical microbiology (DCM) to Statens Serum Institut (SSI), the national public health institute for infectious diseases (www.ssi.dk). Until 2013, all reported data were handled manually before being processed. This system was not flexible and lacked timeliness as well as complete reporting.

In the late 1990s, electronic submission of laboratory reports to general practitioners (GPs) was initiated in Denmark and currently, all communication regarding test results and test ordering between GPs and DCMs (Figure 1) are electronic, using national standard protocols [1]. Electronic reporting from the local DCM to hospitals was initiated on a small scale in 2005 , but has now become widespread. However, the hospital or the GP information systems offer no common facility for sharing pertinent microbiological information between healthcare providers, and this lack of data sharing represents an increasing challenge due to frequent transfer of patients between hospitals.

Based on a pilot project involving two DCMs with different data structure and coding, the SSI took in 2008 the initiative to establish a nationwide database for microbiology in order to enable real-time surveillance of communicable diseases and microorganisms as well as provide nationwide access for healthcare personnel to microbiology reports. By addressing these two aims in one project, the benefit of this new activity was maximised.

\section{Organisation of the MiBa project}

The Danish Microbiology Database (MiBa) project was based on a practical philosophy of data sharing and on a number of core principles:

- MiBa was established by a collaborative effort of all Danish DCMs;

- The DCMs transmit to MiBa a copy of all final electronic reports sent to clinical departments or GPs submitting patient samples for clinical microbiology;

- All data transferred to MiBa from a local DCM remain the property of the local DCM and thus respect local authority and data control;

- Decisions on standardisation of coding and data structure should be made jointly and implemented stepwise respecting local challenges;

- Access to patient reports is only permitted for healthcare providers engaged in diagnosis, treatment or consultation concerning the patient; the statutory national surveillance of infectious diseases is excluded from this clause;

- Access to relevant patient information must be easy, without compromising a high level of data protection;

- Access to data for purposes other than surveillance must comply with Danish law and be approved by the each DCM involved.

The director of the Department of Infectious Disease Epidemiology at the SSI is legally the owner of the $\mathrm{MiBa}$ project and is financially responsible for management and development. The overall steering of $\mathrm{MiBa}$ is undertaken by a board of representatives from each of the 14 DCMs in Denmark and the director who serves as head. The board works in close collaboration with an executive MiBa manager, who from January 2010 has been a medical doctor, specialised in clinical microbiology. Additional staff are a part time software developer and a secretary. Most of the development and continuous upgrading of the MiBa database software has been outsourced to a private supplier of laboratory information systems and funded by the Ministry of Health. 
Electronic communication of microbiological laboratory test orders and reports, Denmark

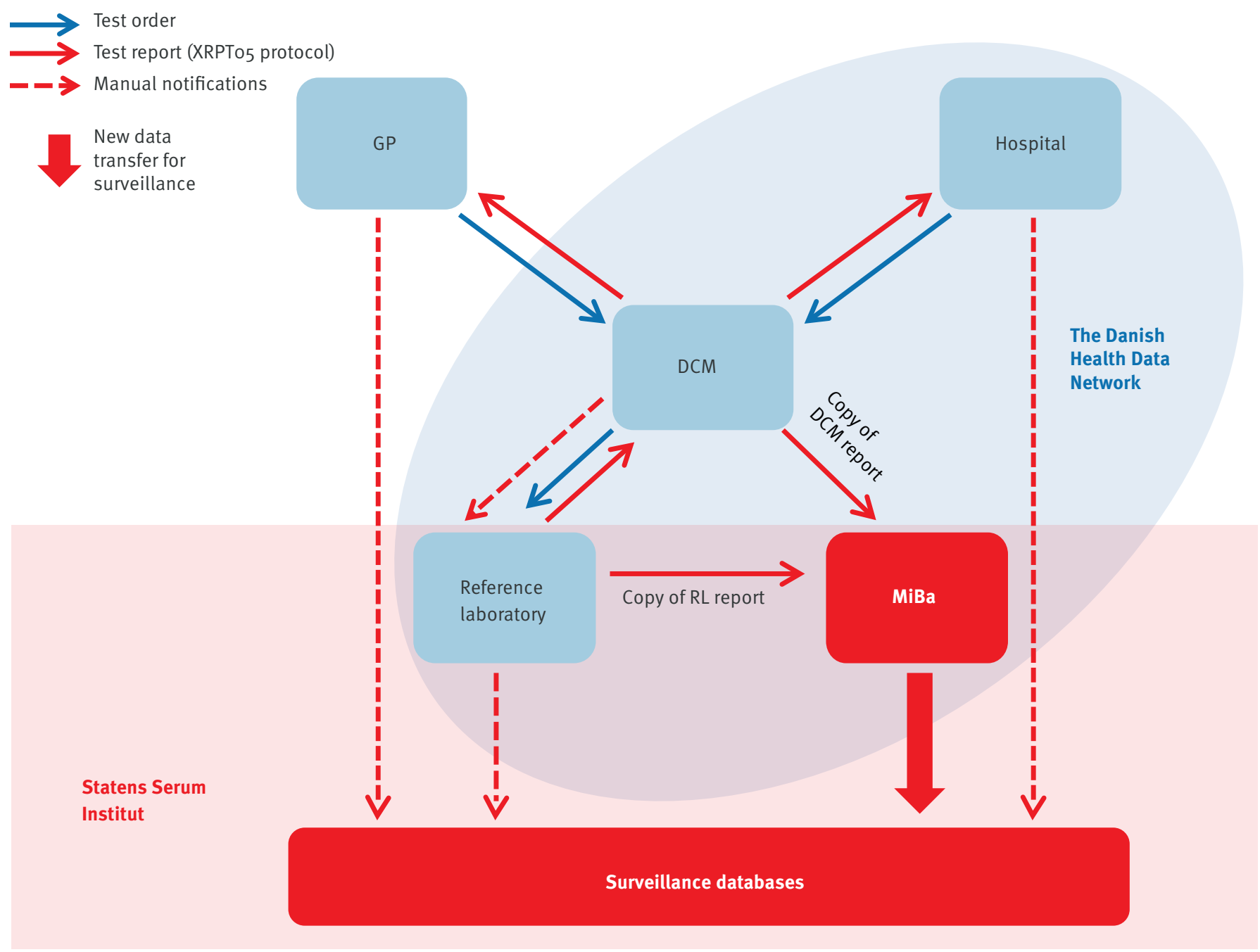

DCM: department of clinical microbiology; GP: general practitioner; MiBa: the Danish Microbiology Database; RL: Reference laboratory; XRTP05: MedCom XRPTo5 $\mathrm{xml}$ data transfer protocol.

Principles of dataflow from the departments of clinical microbiology to the MiBa database

A national standard $\mathrm{xml}$ transfer protocol for microbiology reports (XRPTo5) was issued in 2007 in collaboration with MedCom $[1,2]$. MedCom facilitates the cooperation between authorities, organisations and private companies linked to the Danish healthcare sector, with special focus on solutions for electronic communication. Several different laboratory information systems are in use in Denmark. The protocol enables standardised data output from DCMs with different laboratory information systems and DCM-specific data structure. Data are transferred to MiBa via The Danish Health Data Network which allows the health sector in Denmark to communicate and transfer person-based information to connected organisations through one secure digital solution [1].
The key principle is the simultaneous submission to MiBa of an electronic copy every time a new or updated report is sent from a DCM to the GP or hospital department that requested the test. From the XRPTo5 $\mathrm{xml}$ protocol, the report is imported as a 'local version', i.e. the version available to the healthcare provider (Figures 2 and 3).

Each report is identified by a MiBa identification (ID) number, the patient's unique civil registry number (the CPR number) [3] and the report ID number used by the local DCM. By use of these numbers, reports can be updated if later changes are made by the local DCM. Only the latest version of a report is available in MiBa.

Since January 2010, copies of all reports submitted by Danish DCMs have been transferred to MiBa. By 
Electronic communication of microbiological laboratory test orders and reports, Denmark

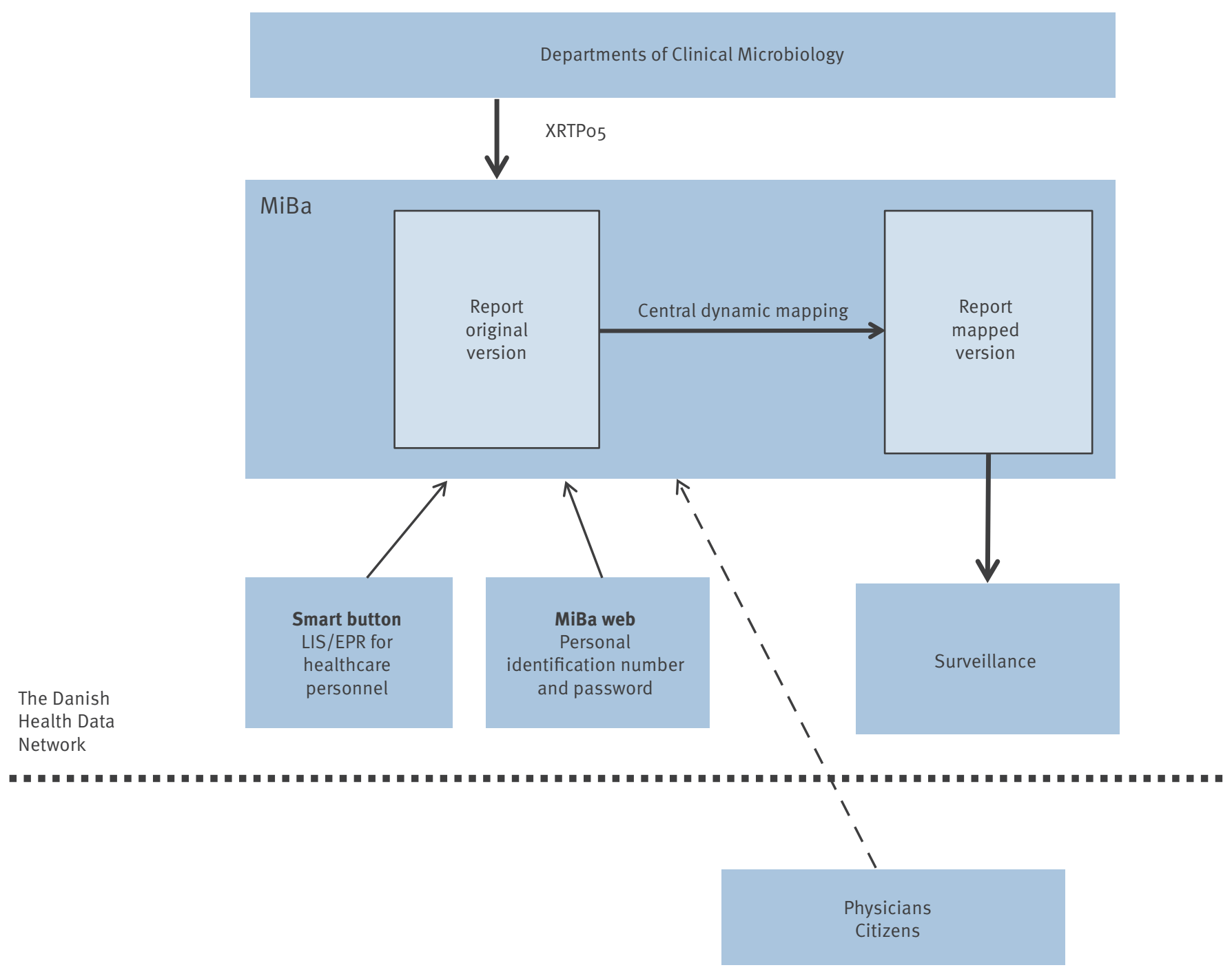

EPR: electronic patient record system; LIS: laboratory information system; MiBa: the Danish Microbiology Database; XRTP05: MedCom XRPTo5 $\mathrm{xml}$ data transfer protocol.

December 2013, approximately 11 million reports had been uploaded.

\section{Access to patient test reports}

Access to the reports in MiBa can be obtained in three different ways (see Figure 2):

- 'MiBa buttons' integrated in local laboratory information systems and electronic patient record systems:

- The healthcare provider has already identified themselves during the procedure for access to the local system. When the MiBa button is pressed, information on the local user ID, the user's organisation and the patient identifier in question are automatically forwarded to MiBa. A new login session is not necessary.
- Login by access from a website using a personal user ID and password:

- A selected group of persons (such as physicians in DCMs, public health personnel) can access MiBa this way, providing more functionality than the MiBa buttons described above. For both solutions, it is a prerequisite that the computer used is covered by a contract with the Danish Health Data Network; this is the case for all hospital computers [1].

- GPs and other healthcare professionals not covered by contracts with the Danish Health Data Network can obtain access to MiBa via a national web portal (www.sundhed.dk). This access requires a digital signature which is a national solution to enable online electronic ID verification on public websites [1]. 
Local version of a laboratory report, Denmark

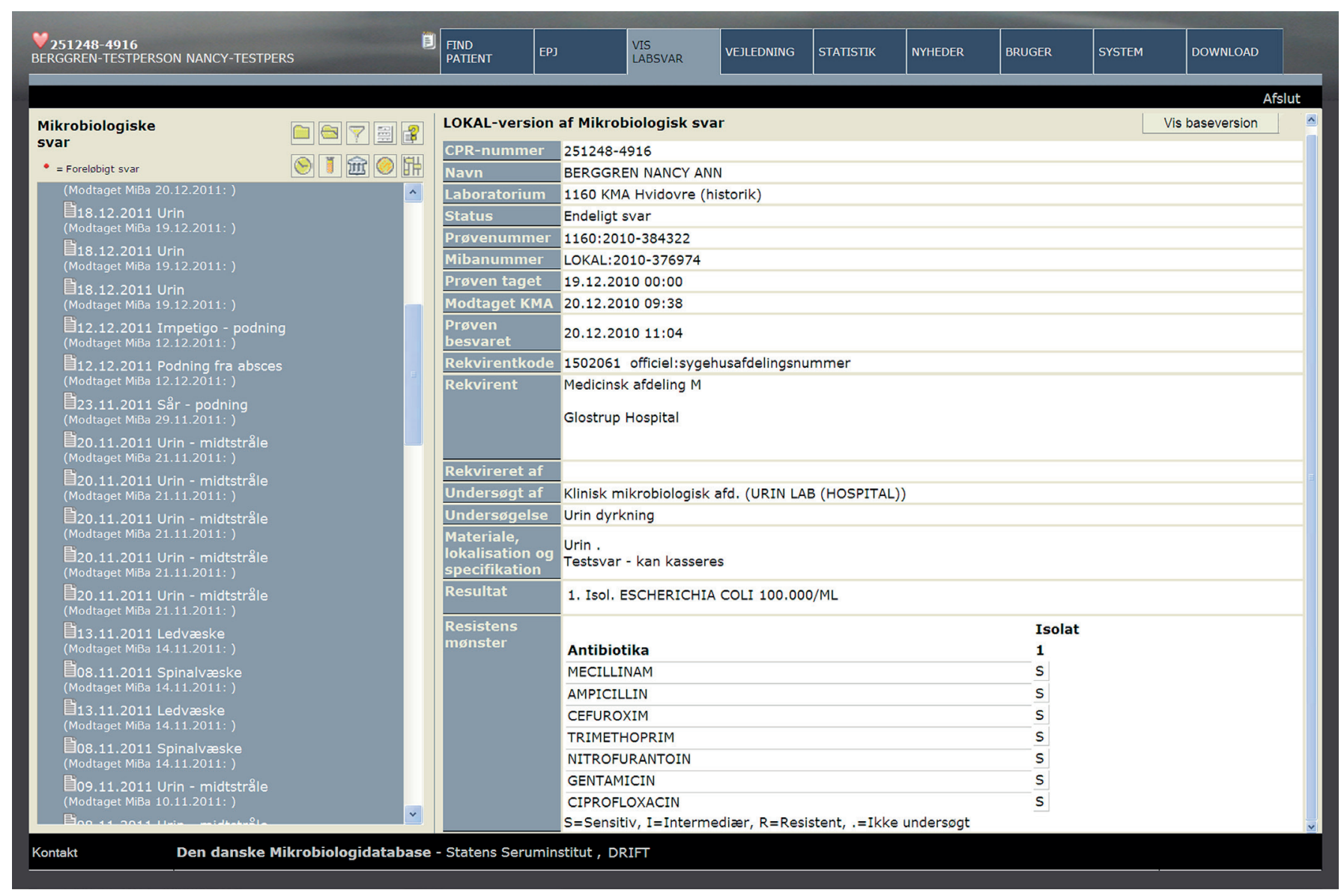

The reports shown are from a fictitious person.

When admitted to hospital patients provide consent for sharing their data within the healthcare system. In rare cases, consent is refused, and these reports are not accessible in MiBa. All activities in MiBa, including user login, are logged and are traceable. Patients do not yet have access to their own microbiology reports. However, we expect that patients in the near future will be able to access their own personal information in MiBa through the national web portal [1]. When access is obtained, an overview of all the patient's reports is shown (Figure 3). Different views and search functions are available. During the first year, access was only obtained by clinical microbiologists, hereafter other healthcare staff were increasingly using the database (Figure 4).

\section{The principle of central dynamic mapping in MiBa}

Although all DCMs provide information like 'microorganism identified' and 'test performed' in coded form, a national standard for coding is not applied for all variables (Box 1). Some elements in the reports follow national coding standards whereas other elements are coded according to local standards. Uniform coding and terminology are required for data extraction and statistics. By use of the function Central Dynamic Mapping, all local codes are mapped to shared codes within MiBa. All reports in MiBa are copied and saved as a 'mapped version', in which all codes have been mapped automatically into shared codes (Figure 1). If the key for mapping between a given local code and the shared code is changed, the code in question is remapped in all uploaded reports (mapped version) from the DCM in question. The local version of the report remains unchanged.

In the MiBa collaboration, we are developing and maintaining shared codes (national terminologies) for reporting laboratory findings. Existing and new working groups have contributed, including a standing committee working on national request codes for detailing sample materials and examinations [4].

\section{Data extraction from $\mathrm{MiBa}$ and national surveillance}

Data extraction from $\mathrm{MiBa}$ is based on the shared codes in the mapped version of the report (Figure 1 ). 


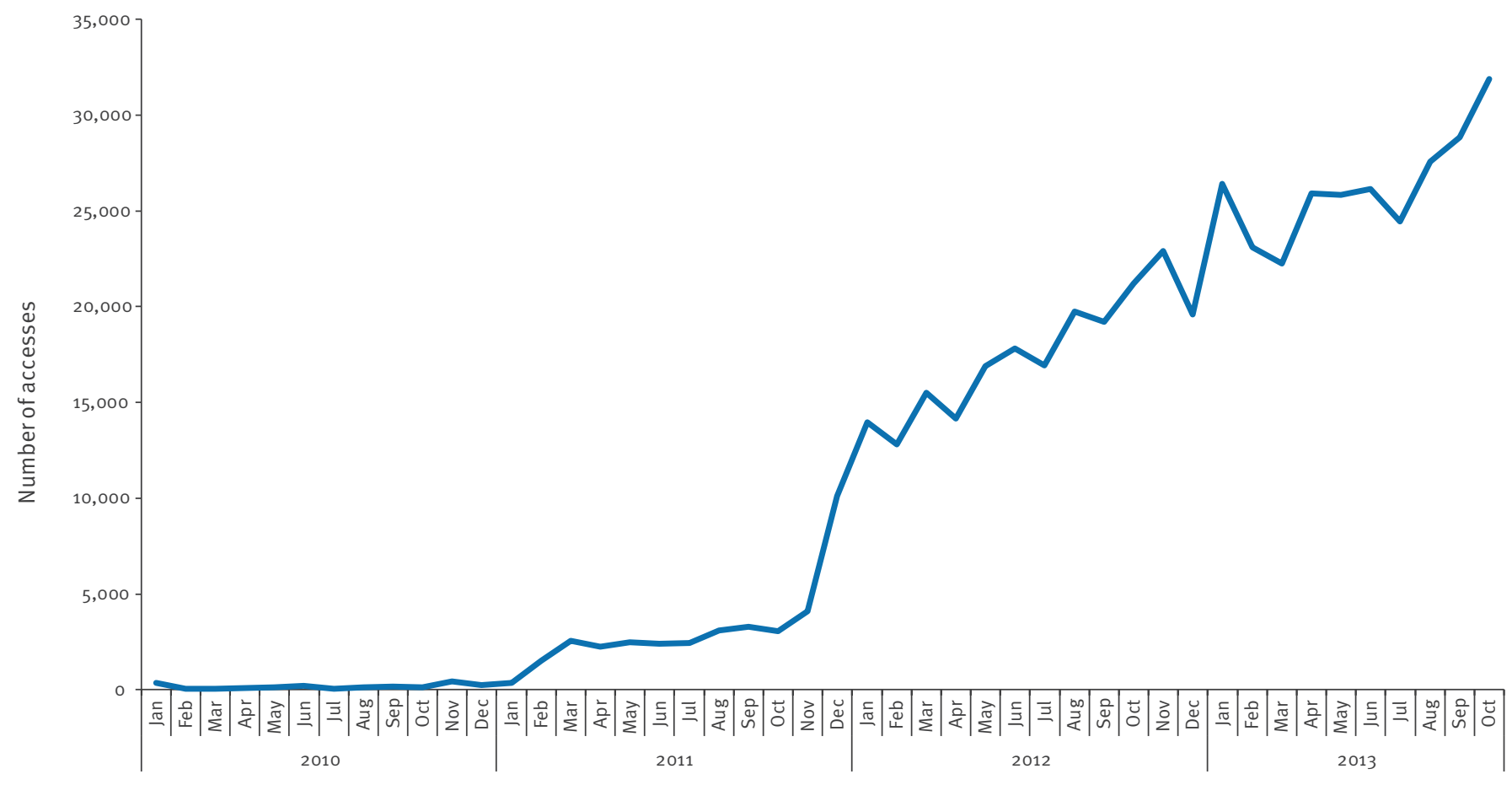

Date

MiBa: the Danish Microbiology Database.

The use of MiBa in nationwide sharing of reports is illustrated in the figure as accesses per month defined as the number of times a healthcare employee looks up reports on a civil registry number (patient identification) per month.

Functionalities are created to specify case definitions and manage automatic transfer of data to specific databases used for laboratory-based surveillance. MiBa can be applied in a real-time workflow to enhance the detection of outbreaks and timely analysis of trends. $\mathrm{MiBa}$ contains negative test results as well, facilitating an understanding of diagnostic practices, which is important in the interpretation of surveillance trends. Examples are given in Box 2. Aggregated and graphical surveillance data will be accessible on an interactive website (http://www.ssi.dk/Smitteberedskab/ Sygdomsovervaagning.aspx). Reports from patients who have refused the sharing of their data among healthcare personnel will still be accessible for data extraction and surveillance.

Individual cases of notifiable diseases are still reported on paper forms by the attending physicians. These forms are often delayed or forgotten. In the future, digital public health surveillance, MiBa-based laboratory surveillance and notifications by clinicians will be integrated in the same workflow in order to provide complete and timely notification to the public health officers at SSI.
MiBa will also serve as a resource for researchers and stimulate standardisation and quality assurance in public health microbiology and public health surveillance [5]. The microbiologist, clinician or researcher cannot extract data themselves. They can only look up records on specified patients, one patient at a time. For data extraction, an application is needed. As data are owned by the local DCMs, approval for projects on MiBa data must be obtained from each individual DCM. In Denmark it is possible to link data across national registries by the CPR number. Data from MiBa and the Danish vaccination register [6] have been linked to estimate vaccine effectiveness [7]. In that study, patients with negative test results were used as controls.

\section{Limitations for the use of MiBa for national surveillance}

A limitation to the current version of MiBa is the lack of standardisation of some types of information (e.g. data on subtyping). The consequence is that key information may be reported as plain text which is not well suited for data extraction.

Another limitation is that the uploaded reports contain information relevant for the treatment of the patient and are targeted for the attending physician and for 
Box 1

Variables in MiBa, Denmark

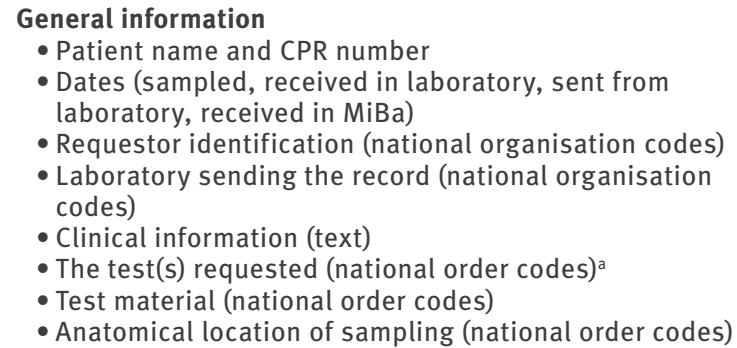

- Dates (sampled, received in laboratory, sent from laboratory, received in MiBa)

- Requestor identification (national organisation codes)

- Laboratory sending the record (national organisation codes)

- Clinical information (text)

- The test(s) requested (national order codes) ${ }^{a}$

- Test material (national order codes)

- Anatomical location of sampling (national order codes)

Specific test (i.e. specific PCR or serological tests)

- The analysis performed (local code)

- Test result

- Interpretation of test result

(i.e. positive or negative)

Complex test (i.e. culture)

- Microorganism(s) found (local code)

- Antimicrobial resistance pattern (local code)

- Microscopy result (text or local code)

\section{Interpretation}

- Overall interpretation of test results (text)

- Comments on test or test results (text)

CPR number: the patient's unique civil registry number; MiBa: the Danish Microbiology Database; PCR: polymerase chain reaction.

a Whether a test is a screening test or a test in disease-related diagnostics, is not registered by a separate variable, but this information may be evident from the order codes used or may be included in the report as a text comment.

inclusion in the patient's health record. Additional data may be needed for surveillance purposes. For instance, information on antimicrobial susceptibility patterns can be restricted in accordance with local antibiotic policies, leaving out information important for surveillance of multidrug-resistant microorganisms.

A new collaborative project has been initiated to overcome the above limitations. This project, which is called the 'subtype project', includes i) construction of a new enlarged $\mathrm{xml}$ transfer protocol that allows for special resistance data (not to be shown for the clinician) and subtyping data to be transferred to MiBa in a standardised manner, ii) establishment of a nationally accepted terminology for subtyping data and iii) implementation of necessary changes in registration procedures in local DCMs.

\section{Lessons learned}

The most important lesson was that a complex healthcare IT-project like MiBa depends on close collaboration between all stakeholders: the DCMs, suppliers of laboratory information systems, clinical users and political decision makers. Goals, concerns and technical possibilities may change over time and development must proceed in manageable iterative steps to accommodate new realities.
It has been a prerequisite that the project received external funding. In addition, the most important barriers to participation were issues of data ownership and authority over their use as well as concerns about extra workload in the local DCMs during phases of development. As soon as it became clear that data transferred to MiBa remained the property of the local DCMs, and that all major decisions on the development of MiBa must be approved by the MiBa board, the collaboration was dynamic and the MiBa project showed steady progress regarding parameters such as number of accesses, completeness of coding, willingness of changing local data structure, development of national codes, number of research projects in progress, etc.

The emphasis on data transmission while accepting local coding, variations in data standards and allowing the DCMs to harmonise data structure and coding in a stepwise manner, is the main reason why MiBa could be established within a short time span of two years. When local DCMs experienced the benefit of sharing microbiology reports, attitudes changed towards sharing even more data. We must emphasise that MiBa would not have been possible without the commitment and support by the local DCMs, which have always been willing to assist in testing and resolving any issues related to internal procedures or codes. However, it needs to be acknowledged that changes in local IT systems and workflows are time-consuming processes and that differences in reporting laboratory diagnostic work may remain.

\section{Achievements and planned activities}

Currently, all physicians have access to microbiological test reports nationwide. All microbiologists, most GPs

\section{Box 2}

Examples of how $\mathrm{MiBa}$ is used in national surveillance, Denmark

\section{The intermittent workflow}

Bordetella pertussis data are extracted every third month (both positive and negative test results).

The variables returned from MiBa are: local record ID, patient CPR number, sex, age, date of sampling, test material, type of test, results of test, ID of the requestor. Information on municipality of residence is obtained from the national CPR register. Using the CPR number, vaccine status can be obtained from the Danish vaccine registry (3). A detailed report is sent back to the DCM.

\section{The continuous workflow}

An automatic data transfer is established between a prototype of the National Registry of Enteric Pathogens and MiBa.

Once a day, MiBa returns all new records, in which an enteric pathogen has been found. The number of episodes are calculated and updated every day based on CPR number, the type of pathogen found and the time interval between records.

CPR number: the patient's unique civil registry number; DCM: department of clinical microbiology; ID: identification number; MiBa: the Danish Microbiology Database. 
and around one third of clinicians in hospitals have smart-button access solutions.

Laboratory surveillance for pertussis and influenza are now fully based on MiBa data. An electronic weekly report on influenza surveillance was launched in the winter 2012/13 with free access (http://www.ssi.dk/ Aktuelt/Nyhedsbreve/INFLUENZA-NYT.aspx).

For validation purposes, MiBa-based surveillance of a number of microorganisms is currently running in parallel with the existing reporting system. In the spring of 2014, surveillance of individual microorganisms will one by one be changed to the MiBa system only after successful validation.

Planned activities include:

- development and implementation of the 'subtype project',

- development of electronic workflows integrating laboratory-based surveillance with notifications from the attending physicians for selected diseases under statutory surveillance,

- provision of smart access solutions to all hospital physicians,

- provision of access for patients to their own reports.

\section{Conclusions}

One of the visions for the healthcare system in Denmark is to provide access to test results across the entire health sector, which is vital for the development of secure, efficient work processes and high standards of public health surveillance [1]. MiBa meets this vision. The development of MiBa emerged from a unique collaborative environment including all Danish DCMs, the Department of Infectious Disease Epidemiology, the suppliers of laboratory information systems and MedCom. Development of MiBa with a limited budget and within a short time has only been possible because all stakeholders, including laboratory information system suppliers, were personally engaged.

The nationwide sharing of real-time microbiological reports has been a success. The integration of $\mathrm{MiBa}$ in the national eHealth infrastructure is still being improved. Automatic workflows for laboratory surveillance are in progress. The large benefits of a timely and complete surveillance system are still to be experienced, but prototype examples show promising results. Finally, MiBa will provide numerous opportunities as a data source for national surveillance and research projects.

MiBa Board of representatives

Bettina Lundgren, Centre of Diagnostic Investigation, Rigshospitalet, Copenhagen; Christian Østergaard Andersen, DCM, Hvidovre Hospital; Jens Otto Jarløv and Dennis Schrøder Hansen, DCM Herlev and Hillerød Hospital; Michael Tvede, DCM, Rigshospitalet; Henrik C. Schønheyder, DCM, Aalborg University Hospital; Thøger Gorm Jensen, DCM, Odense University Hospital; Ram Dessau, DCM,
Slagelse Hospital; Jens K. Møller, DCM, Vejle Hospital; Svend Ellermann-Eriksen, DCM, Skejby, Aarhus University Hospital; Steen Lomborg and Jørgen Prag, DCM Central-West Region; Poul Kjældgaard, DCM Sønderborg Hospital; Kjeld Truberg, DCM, Esbjerg Hospital; Steen Hoffmann, Statens Serum Institut.

\section{Acknowledgements}

The Danish Microbiology Database is funded by the Danish Ministry of Health.

Authors' contributions

$M$ Voldstedlund has drafted the first version, made revisions, and approved the final version of the paper; has been managing MiBa science 2010. K Mølbak revised the first draft of the paper critically and approved the final version; has established MiBa. M Haahr has been engaged in the MiBa project, contributed to the illustrations, and approved the final version of the paper. The MiBa board of representatives have revised the first draft of the paper and approved the final version; each member has been part of the collaborative effort establishing MiBa.

\section{References}

1. MedCom. eHealth in Denmark. eHealth as a part of a coherent Danish health care system. Copenhagen: The Danish Ministry of Health; 2012. ISBN 978-87-7601-332-5, Available from: http://www.medcom.dk/dwn5350

2. Det nye gode XML mikrobiologisvar. [The good new XML microbiology reports]. Odense: MedCom; June 2011. Danish. Available from: http://svn.medcom.dk/svn/releases/ Standarder/Det\%20gode\%2omikrobiologiwebsvar/XRPT05.pdf

3. Pedersen CB. The Danish civil registration system. Scand J Public Health. 2011;39 (7 Suppl):22-5. http://dx.doi.org/10.1177/1403494810387965 PMid:21775345

4. Møller JK, Jensen TG, Prag J, Tønning B, Pedersen S, Jarløv $\mathrm{JO}$, et al. Elektronisk svar og rekvisition i klinisk mikrobiologi - Kodeværk for prøvebeskrivelse. [Electronic reporting and requisition in clinical microbiology - Codes for the description of samples]. Danish Society of Clinical Microbiology; 2002. In Danish. Available from: http://www.dskm.dk/pdf/rapporter/ DSKM-rapporter/MDS_Rapport.pdf

5. Frank L. When an entire country is a cohort. Science. 2000;287(5462):2398-9.

http://dx.doi.org/10.1126/science.287.5462.2398 PMid:10766613

6. Grove Krause T, Jakobsen S, Haarh M, Mølbak K. The Danish vaccination register. Euro Surveill. 2012;17(17): $\mathrm{pii}=20155$. Available from: http://www.eurosurveillance.org/ViewArticle. aspx?Articleld $=20155$ PMid:22551494

7. Bragstad K, Emborg HD, Kølsen Fischer T, Voldstedlund M, Gubbels S, Andersen B, et al. Low vaccine effectiveness against influenza $A\left(\mathrm{H}_{3} \mathrm{~N}_{2}\right)$ virus among elderly people in Denmark in 2012/13 - a rapid epidemiological and virological assessment. Euro Surveill. 2013;18(6):pii=20397. Available from: http://www.eurosurveillance.org/ViewArticle. aspx?Articleld $=20397$ 